COMMISSIONS G1 AND G4 OF THE IAU

INFORMATION BULLETIN ON VARIABLE STARS

Volume 63 Number 6219 DOI: 10.22444/IBVS.6219

Konkoly Observatory

Budapest

11 October 2017

HU ISSN $0374-0676$

\title{
TIMES OF MINIMA OF SOME ECLIPSING BINARY STARS WITH ECCENTRIC ORBIT IN THE KEPLER FIELD
}

BULUT, ì.

Department of Space Sciences and Technologies, Faculty of Arts and Sciences, Çanakkale Onsekiz Mart University, Terzioğlu Kampüsü, TR-17020, Çanakkale, Turkey; e-mail: ibulut@comu.edu.tr

\section{Observatory and telescope:}

The Kepler photometer is a Schmidt telescope design with a 0.95-meter aperture and a 105 square deg (about 12 degree diameter) FOV.

\begin{tabular}{|l|l|}
\hline Detector: & The photometer camera contains 42 CCDs with \\
& $2200 \times 1024$ pixels, where each pixel covers 4 arcsec.
\end{tabular}

\begin{tabular}{|l|lllll|}
\hline Method of data reduction: & & & & & \\
\hline $\begin{array}{l}\text { Photometry flux values were taken from the } \\
\text { (http://keplerebs.villanova.edu) }\end{array}$ & & & & & \\
\hline
\end{tabular}

\section{Method of minimum determination:}

The minima times were computed with the Kwee-van Woerden method (Kwee \& van Woerden, 1956).

\section{Remarks:}

We present 517 minima times of 6 eclipsing binaries with eccentric orbit. The $\mathrm{O}-\mathrm{C}$ diagrams are shown in Figs. 1 and 2.

\section{Acknowledgements:}

This paper includes data collected by the Kepler mission. Funding for the Kepler mission is provided by the NASA Science Mission directorate.

Reference:

Kwee, K. K., van Woerden, H., 1956, Bull. Astron. Inst. Netherlands, 12, 327 


\begin{tabular}{|ccccc|}
\hline Times of minima: & & & \\
\hline Star name & Time of min. & Error & Type & Filter \\
& HJD 2400000+ & & & \\
\hline KIC 4932691 & 54967.70418 & 0.00299 & I & Kepler \\
& 54972.50630 & 0.01486 & II & Kepler \\
& 54985.83524 & 0.00524 & I & Kepler \\
54990.47957 & 0.01034 & II & Kepler \\
55003.94523 & 0.00328 & I & Kepler \\
55022.05529 & 0.00454 & I & Kepler \\
55026.68879 & 0.00806 & II & Kepler \\
55040.16346 & 0.00549 & I & Kepler \\
55045.01905 & 0.00993 & II & Kepler \\
55058.27043 & 0.00400 & I & Kepler \\
55063.18681 & 0.01446 & II & Kepler \\
55076.39419 & 0.00323 & I & Kepler \\
55081.19915 & 0.01471 & II & Kepler \\
55094.49652 & 0.00504 & I & Kepler \\
55099.45777 & 0.01025 & II & Kepler \\
55112.60843 & 0.00424 & I & Kepler \\
55117.60643 & 0.02323 & II & Kepler \\
55130.71894 & 0.00427 & I & Kepler \\
55135.39270 & 0.00551 & II & Kepler \\
55148.84864 & 0.00595 & I & Kepler \\
55153.62809 & 0.01108 & II & Kepler \\
55166.94809 & 0.00349 & I & Kepler \\
55171.67254 & 0.01557 & II & Kepler \\
55189.85773 & 0.00559 & II & Kepler \\
55203.16352 & 0.00605 & I & Kepler \\
55207.99029 & 0.00639 & II & Kepler \\
55221.28102 & 0.00293 & I & Kepler \\
55226.06370 & 0.00618 & II & Kepler \\
55239.38710 & 0.00558 & I & Kepler \\
55244.10342 & 0.00597 & II & Kepler \\
55257.49469 & 0.00599 & I & Kepler \\
55262.31028 & 0.02012 & II & Kepler \\
55280.43424 & 0.00866 & II & Kepler \\
55293.72957 & 0.00471 & I & Kepler \\
55298.72648 & 0.00948 & II & Kepler \\
55311.84560 & 0.00334 & I & Kepler \\
55316.80536 & 0.00950 & II & Kepler \\
55329.94437 & 0.00366 & I & Kepler \\
55334.87176 & 0.01468 & II & Kepler \\
55348.05019 & 0.00516 & I & Kepler \\
55352.87147 & 0.00717 & II & Kepler \\
& 55370.97493 & 0.00031 & II & Kepler \\
\hline
\end{tabular}




\begin{tabular}{|llccl|}
\hline Times of minima: & & & \\
\hline Star name & Time of min. & Error & Type & Filter \\
& HJD 2400000+ & & & \\
\hline KIC 4932691 & 55474.85393 & 0.00359 & I & Kepler \\
& 55492.96744 & 0.00488 & I & Kepler \\
55511.07491 & 0.00368 & I & Kepler \\
55515.69518 & 0.02536 & II & Kepler \\
55529.18751 & 0.00416 & I & Kepler \\
55534.01506 & 0.01239 & II & Kepler \\
55547.30372 & 0.00432 & I & Kepler \\
55552.09953 & 0.00136 & II & Kepler \\
55583.51992 & 0.00457 & I & Kepler \\
55588.30124 & 0.00774 & II & Kepler \\
55601.62815 & 0.00555 & I & Kepler \\
55606.45068 & 0.01115 & II & Kepler \\
55619.74491 & 0.00372 & I & Kepler \\
55624.54875 & 0.02524 & II & Kepler \\
55642.68012 & 0.01251 & II & Kepler \\
55655.97070 & 0.00422 & I & Kepler \\
55660.77593 & 0.00613 & II & Kepler \\
55674.07624 & 0.00336 & I & Kepler \\
55678.92482 & 0.01446 & II & Kepler \\
55692.20751 & 0.00368 & I & Kepler \\
55696.83902 & 0.00769 & II & Kepler \\
55710.31997 & 0.00518 & I & Kepler \\
55715.11061 & 0.00864 & II & Kepler \\
55728.40921 & 0.00580 & I & Kepler \\
55733.10453 & 0.00870 & II & Kepler \\
55837.09629 & 0.00336 & I & Kepler \\
55841.73208 & 0.00782 & II & Kepler \\
55855.20150 & 0.00505 & I & Kepler \\
55873.30815 & 0.00616 & I & Kepler \\
55878.10419 & 0.01676 & II & Kepler \\
55891.43983 & 0.00356 & I & Kepler \\
55896.13096 & 0.02619 & II & Kepler \\
55909.55387 & 0.00607 & I & Kepler \\
55914.34397 & 0.01942 & II & Kepler \\
55927.65944 & 0.00313 & I & Kepler \\
55932.41110 & 0.01621 & II & Kepler \\
55945.75909 & 0.00347 & I & Kepler \\
55963.87715 & 0.00344 & I & Kepler \\
55968.71817 & 0.01174 & II & Kepler \\
55981.99129 & 0.00260 & I & Kepler \\
& 5596.79681 & 0.00897 & II & Kepler \\
& 0.00399 & I & Kepler \\
& & & &
\end{tabular}




\begin{tabular}{|c|c|c|c|c|}
\hline \multicolumn{5}{|c|}{ Times of minima: } \\
\hline Star name & $\begin{array}{l}\text { Time of min. } \\
\text { HJD } 2400000+\end{array}$ & Error & Type & Filter Rem. \\
\hline \multirow[t]{30}{*}{ KIC 4932691} & 56018.21168 & 0.00531 & $\mathrm{I}$ & Kepler \\
\hline & 56023.10999 & 0.00719 & II & Kepler \\
\hline & 56036.33259 & 0.00312 & $\mathrm{I}$ & Kepler \\
\hline & 56054.43580 & 0.00485 & $\mathrm{I}$ & Kepler \\
\hline & 56059.19200 & 0.00648 & II & Kepler \\
\hline & 56072.54284 & 0.00304 & $\mathrm{I}$ & Kepler \\
\hline & 56077.39264 & 0.02352 & II & Kepler \\
\hline & 56090.66130 & 0.00502 & $\mathrm{I}$ & Kepler \\
\hline & 56217.43251 & 0.00638 & $\mathrm{I}$ & Kepler \\
\hline & 56222.10179 & 0.01635 & II & Kepler \\
\hline & 56235.54363 & 0.00497 & $\mathrm{I}$ & Kepler \\
\hline & 56240.20381 & 0.00983 & II & Kepler \\
\hline & 56253.68256 & 0.00493 & $\mathrm{I}$ & Kepler \\
\hline & 56258.30120 & 0.01005 & II & Kepler \\
\hline & 56271.78818 & 0.00361 & $\mathrm{I}$ & Kepler \\
\hline & 56276.60675 & 0.01129 & II & Kepler \\
\hline & 56289.89729 & 0.00528 & $\mathrm{I}$ & Kepler \\
\hline & 56308.01162 & 0.00326 & $\mathrm{I}$ & Kepler \\
\hline & 56326.11999 & 0.00354 & $\mathrm{I}$ & Kepler \\
\hline & 56330.77953 & 0.01279 & II & Kepler \\
\hline & 56344.23598 & 0.00281 & I & Kepler \\
\hline & 56349.00703 & 0.00778 & II & Kepler \\
\hline & 56362.34323 & 0.00628 & I & Kepler \\
\hline & 56367.23512 & 0.00901 & II & Kepler \\
\hline & 56380.45960 & 0.00431 & $\mathrm{I}$ & Kepler \\
\hline & 56385.17812 & 0.00408 & II & Kepler \\
\hline & 56398.57000 & 0.00383 & I & Kepler \\
\hline & 56403.50721 & 0.01102 & II & Kepler \\
\hline & 56421.35880 & 0.00662 & $\mathrm{I}$ & Kepler \\
\hline & 56421.35880 & 0.00662 & II & Kepler \\
\hline \multirow[t]{13}{*}{ KIC 5986209} & 55193.64842 & 0.00072 & II & Kepler \\
\hline & 55202.42103 & 0.00023 & I & Kepler \\
\hline & 55217.18138 & 0.01519 & II & Kepler \\
\hline & 55226.15876 & 0.00028 & I & Kepler \\
\hline & 55241.12402 & 0.00038 & II & Kepler \\
\hline & 55249.89820 & 0.00045 & I & Kepler \\
\hline & 55264.86249 & 0.00036 & II & Kepler \\
\hline & 55273.63445 & 0.00046 & $\mathrm{I}$ & Kepler \\
\hline & 55288.60083 & 0.00038 & II & Kepler \\
\hline & 55297.37304 & 0.00054 & $\mathrm{I}$ & Kepler \\
\hline & 55312.33872 & 0.00033 & II & Kepler \\
\hline & 55321.11099 & 0.00019 & I & Kepler \\
\hline & 55336.07588 & 0.00042 & II & Kepler \\
\hline
\end{tabular}




\begin{tabular}{|ccccl|}
\hline Times of minima: & & & \\
\hline Star name & Time of min. & Error & Type & Filter \\
& HJD 2400000+ & & & \\
\hline KIC 5986209. & 55344.84860 & 0.00038 & I & Kepler \\
& 55359.81417 & 0.00025 & II & Kepler \\
55368.58758 & 0.00028 & I & Kepler \\
55383.55213 & 0.00024 & II & Kepler \\
55392.32588 & 0.00044 & I & Kepler \\
55407.29028 & 0.00022 & II & Kepler \\
55416.06252 & 0.00042 & I & Kepler \\
55431.02708 & 0.00051 & II & Kepler \\
55439.80156 & 0.00052 & I & Kepler \\
55454.76593 & 0.00030 & II & Kepler \\
55463.53828 & 0.00039 & I & Kepler \\
55478.50369 & 0.00035 & II & Kepler \\
55487.27696 & 0.00007 & I & Kepler \\
55502.24210 & 0.00026 & II & Kepler \\
55511.01443 & 0.00038 & I & Kepler \\
55525.97983 & 0.00031 & II & Kepler \\
55534.75278 & 0.00010 & I & Kepler \\
55549.71805 & 0.00027 & II & Kepler \\
55573.45558 & 0.00022 & II & Kepler \\
55582.22885 & 0.00015 & I & Kepler \\
55597.19444 & 0.00087 & II & Kepler \\
55605.96609 & 0.00039 & I & Kepler \\
55620.93123 & 0.00091 & II & Kepler \\
55629.70525 & 0.00011 & I & Kepler \\
55644.66989 & 0.00033 & II & Kepler \\
55653.44196 & 0.00024 & I & Kepler \\
55668.40751 & 0.00035 & II & Kepler \\
55677.18123 & 0.00027 & I & Kepler \\
55692.14564 & 0.00030 & II & Kepler \\
55700.91868 & 0.00007 & I & Kepler \\
55715.88389 & 0.00042 & II & Kepler \\
55724.65616 & 0.00023 & I & Kepler \\
55748.39506 & 0.00020 & I & Kepler \\
55763.35897 & 0.00026 & II & Kepler \\
55772.13268 & 0.00009 & I & Kepler \\
55787.09711 & 0.00038 & II & Kepler \\
55795.87040 & 0.00031 & I & Kepler \\
55810.83502 & 0.00039 & II & Kepler \\
55819.60878 & 0.00010 & I & Kepler \\
55834.57295 & 0.00042 & II & Kepler \\
55843.34611 & 0.00026 & I & Kepler \\
& 55867.08432 & 0.00061 & I & Kepler \\
\hline
\end{tabular}




\begin{tabular}{|c|c|c|c|c|}
\hline \multicolumn{5}{|c|}{ Times of minima: } \\
\hline Star name & $\begin{array}{l}\text { Time of min. } \\
\text { HJD } 2400000+\end{array}$ & Error & Type & Filter Rem. \\
\hline \multirow{41}{*}{ KIC 5986209} & 55882.04886 & 0.00033 & II & Kepler \\
\hline & 55890.82177 & 0.00010 & $\mathrm{I}$ & Kepler \\
\hline & 55905.78780 & 0.00056 & II & Kepler \\
\hline & 55914.56047 & 0.00006 & $\mathrm{I}$ & Kepler \\
\hline & 55929.52421 & 0.00032 & II & Kepler \\
\hline & 55938.29767 & 0.00033 & $\mathrm{I}$ & Kepler \\
\hline & 55953.26292 & 0.00067 & II & Kepler \\
\hline & 55962.03550 & 0.00053 & $\mathrm{I}$ & Kepler \\
\hline & 55977.00037 & 0.00031 & II & Kepler \\
\hline & 55985.77614 & 0.00049 & $\mathrm{I}$ & Kepler \\
\hline & 56000.73887 & 0.00022 & II & Kepler \\
\hline & 56009.51276 & 0.00029 & $\mathrm{I}$ & Kepler \\
\hline & 56024.47691 & 0.00028 & II & Kepler \\
\hline & 56033.25087 & 0.00051 & $\mathrm{I}$ & Kepler \\
\hline & 56056.98894 & 0.00031 & $\mathrm{I}$ & Kepler \\
\hline & 56071.95244 & 0.00029 & II & Kepler \\
\hline & 56080.72664 & 0.00026 & $\mathrm{I}$ & Kepler \\
\hline & 56095.69001 & 0.00025 & II & Kepler \\
\hline & 56104.46506 & 0.00063 & $\mathrm{I}$ & Kepler \\
\hline & 56119.42874 & 0.00023 & II & Kepler \\
\hline & 56143.16609 & 0.00032 & II & Kepler \\
\hline & 56151.93970 & 0.00053 & I & Kepler \\
\hline & 56166.90401 & 0.00038 & II & Kepler \\
\hline & 56175.67870 & 0.00030 & $\mathrm{I}$ & Kepler \\
\hline & 56190.64219 & 0.00041 & II & Kepler \\
\hline & 56199.41685 & 0.00041 & $\mathrm{I}$ & Kepler \\
\hline & 56214.37976 & 0.00031 & II & Kepler \\
\hline & 56223.15481 & 0.00048 & $\mathrm{I}$ & Kepler \\
\hline & 56261.85591 & 0.00030 & II & Kepler \\
\hline & 56270.63023 & 0.00060 & $\mathrm{I}$ & Kepler \\
\hline & 56285.59425 & 0.00049 & II & Kepler \\
\hline & 56294.36815 & 0.00013 & I & Kepler \\
\hline & 56309.33150 & 0.00039 & II & Kepler \\
\hline & 56333.06980 & 0.00023 & II & Kepler \\
\hline & 56341.84415 & 0.00015 & I & Kepler \\
\hline & 56356.80821 & 0.00036 & II & Kepler \\
\hline & 56365.58226 & 0.00048 & $\mathrm{I}$ & Kepler \\
\hline & 56380.54538 & 0.00045 & II & Kepler \\
\hline & 56389.32060 & 0.00030 & $\mathrm{I}$ & Kepler \\
\hline & 56404.28350 & 0.00026 & II & Kepler \\
\hline & 56413.05801 & 0.00019 & I & Kepler \\
\hline \multirow[t]{2}{*}{ KIC 6841577} & 54973.27350 & 0.00026 & I & Kepler \\
\hline & 54979.87424 & 0.00091 & II & Kepler \\
\hline
\end{tabular}




\begin{tabular}{|c|c|c|c|c|}
\hline \multicolumn{5}{|c|}{ Times of minima: } \\
\hline Star name & $\begin{array}{l}\text { Time of min. } \\
\text { HJD 2400000+ }\end{array}$ & Error & Type & Filter Rem. \\
\hline \multirow[t]{43}{*}{ KIC 6841577} & 54988.81127 & 0.00043 & $\mathrm{I}$ & Kepler \\
\hline & 54995.41256 & 0.00086 & II & Kepler \\
\hline & 55004.34869 & 0.00052 & I & Kepler \\
\hline & 55010.94840 & 0.00116 & II & Kepler \\
\hline & 55019.88608 & 0.00010 & $\mathrm{I}$ & Kepler \\
\hline & 55026.48648 & 0.00093 & II & Kepler \\
\hline & 55035.42383 & 0.00031 & $\mathrm{I}$ & Kepler \\
\hline & 55042.02373 & 0.00105 & II & Kepler \\
\hline & 55050.96124 & 0.00043 & $\mathrm{I}$ & Kepler \\
\hline & 55057.56337 & 0.00103 & II & Kepler \\
\hline & 55066.49848 & 0.00009 & $\mathrm{I}$ & Kepler \\
\hline & 55073.09922 & 0.00110 & II & Kepler \\
\hline & 55082.03628 & 0.00012 & I & Kepler \\
\hline & 55097.57373 & 0.00021 & $\mathrm{I}$ & Kepler \\
\hline & 55104.17270 & 0.00092 & II & Kepler \\
\hline & 55113.11094 & 0.00049 & $\mathrm{I}$ & Kepler \\
\hline & 55119.71237 & 0.00097 & II & Kepler \\
\hline & 55128.64919 & 0.00033 & I & Kepler \\
\hline & 55135.25101 & 0.00110 & II & Kepler \\
\hline & 55144.18666 & 0.00038 & $\mathrm{I}$ & Kepler \\
\hline & 55150.78804 & 0.00103 & II & Kepler \\
\hline & 55159.72407 & 0.00030 & $\mathrm{I}$ & Kepler \\
\hline & 55166.32461 & 0.00104 & II & Kepler \\
\hline & 55175.26170 & 0.00008 & $\mathrm{I}$ & Kepler \\
\hline & 55181.86028 & 0.00339 & II & Kepler \\
\hline & 55190.79861 & 0.00040 & I & Kepler \\
\hline & 55197.39878 & 0.00088 & II & Kepler \\
\hline & 55206.33649 & 0.00030 & $\mathrm{I}$ & Kepler \\
\hline & 55212.93796 & 0.00103 & II & Kepler \\
\hline & 55221.87432 & 0.00034 & I & Kepler \\
\hline & 55228.47462 & 0.00112 & II & Kepler \\
\hline & 55237.41173 & 0.00036 & $\mathrm{I}$ & Kepler \\
\hline & 55244.01205 & 0.00075 & II & Kepler \\
\hline & 55252.94924 & 0.00009 & $\mathrm{I}$ & Kepler \\
\hline & 55259.54996 & 0.00090 & II & Kepler \\
\hline & 55268.48621 & 0.00033 & I & Kepler \\
\hline & 55377.24962 & 0.00017 & $\mathrm{I}$ & Kepler \\
\hline & 55383.84985 & 0.00128 & II & Kepler \\
\hline & 55392.78717 & 0.00029 & I & Kepler \\
\hline & 55399.38414 & 0.00188 & II & Kepler \\
\hline & 55408.32464 & 0.00019 & I & Kepler \\
\hline & 55414.92481 & 0.00097 & II & Kepler \\
\hline & 55423.86193 & 0.00008 & I & Kepler \\
\hline
\end{tabular}




\begin{tabular}{|ccccc|}
\hline Times of minima: & & & \\
\hline Star name & Time of min. & Error & Type & Filter \\
& HJD 2400000+ & & & \\
\hline KIC 6841577 & 55430.46256 & 0.00254 & II & Kepler \\
& 55439.39929 & 0.00046 & I & Kepler \\
55446.00168 & 0.00112 & II & Kepler \\
55454.93651 & 0.00035 & I & Kepler \\
55461.53810 & 0.00226 & II & Kepler \\
55470.47490 & 0.00027 & I & Kepler \\
55477.07542 & 0.00147 & II & Kepler \\
55486.01292 & 0.00038 & I & Kepler \\
55492.61333 & 0.00298 & II & Kepler \\
55501.54945 & 0.00018 & I & Kepler \\
55508.15193 & 0.00262 & II & Kepler \\
55517.08701 & 0.00011 & I & Kepler \\
55532.62455 & 0.00045 & I & Kepler \\
55539.22506 & 0.00094 & II & Kepler \\
55548.16199 & 0.00043 & I & Kepler \\
55570.30184 & 0.00101 & II & Kepler \\
55579.23756 & 0.00034 & I & Kepler \\
55585.83899 & 0.00086 & II & Kepler \\
55601.37630 & 0.00078 & II & Kepler \\
55610.31234 & 0.00013 & I & Kepler \\
55616.91384 & 0.00180 & II & Kepler \\
55625.84973 & 0.00027 & I & Kepler \\
55632.45125 & 0.00122 & II & Kepler \\
55741.21313 & 0.00096 & II & Kepler \\
55750.15053 & 0.00027 & I & Kepler \\
55756.75042 & 0.00101 & II & Kepler \\
55765.68815 & 0.00045 & I & Kepler \\
55772.28940 & 0.00317 & II & Kepler \\
55781.22526 & 0.00050 & I & Kepler \\
55787.82610 & 0.00167 & II & Kepler \\
55796.76263 & 0.00044 & I & Kepler \\
55803.36537 & 0.00194 & II & Kepler \\
55812.30057 & 0.00021 & I & Kepler \\
55818.90180 & 0.00095 & II & Kepler \\
55827.83814 & 0.00029 & I & Kepler \\
55834.44114 & 0.00155 & II & Kepler \\
55843.37558 & 0.00038 & I & Kepler \\
55849.97538 & 0.00096 & II & Kepler \\
55858.91270 & 0.00012 & I & Kepler \\
55874.45044 & 0.00012 & I & Kepler \\
55881.05156 & 0.00176 & II & Kepler \\
& 55912.12557 & 0.00104 & II & Kepler \\
\hline
\end{tabular}




\begin{tabular}{|llccl|}
\hline Times of minima: & & & \\
\hline Star name & Time of min. & Error & Type & Filter \\
& HJD 2400000+ & & & \\
\hline KIC 6841577 & 55921.06331 & 0.00028 & I & Kepler \\
& 55927.66399 & 0.00173 & II & Kepler \\
& 56107.51330 & 0.00016 & I & Kepler \\
56114.11380 & 0.00105 & II & Kepler \\
56129.65574 & 0.00090 & II & Kepler \\
56145.18845 & 0.00091 & II & Kepler \\
56154.12569 & 0.00043 & I & Kepler \\
56160.72642 & 0.00099 & II & Kepler \\
56176.26457 & 0.00102 & II & Kepler \\
56185.20132 & 0.00031 & I & Kepler \\
56191.80164 & 0.00099 & II & Kepler \\
56200.73849 & 0.00010 & I & Kepler \\
56207.34096 & 0.00178 & II & Kepler \\
56216.27584 & 0.00016 & I & Kepler \\
56222.87201 & 0.00113 & II & Kepler \\
56231.81354 & 0.00048 & I & Kepler \\
56238.42053 & 0.00155 & II & Kepler \\
56253.95181 & 0.00104 & II & Kepler \\
56262.88836 & 0.00035 & I & Kepler \\
56269.49274 & 0.00270 & II & Kepler \\
56278.42657 & 0.00030 & I & Kepler \\
56285.02669 & 0.00081 & II & Kepler \\
56293.96400 & 0.00036 & I & Kepler \\
56300.56403 & 0.00163 & II & Kepler \\
56309.50621 & 0.00081 & I & Kepler \\
56325.03913 & 0.00044 & I & Kepler \\
56331.63855 & 0.00120 & II & Kepler \\
56340.57660 & 0.00041 & I & Kepler \\
56347.17610 & 0.00098 & II & Kepler \\
56356.11448 & 0.00036 & I & Kepler \\
56362.71391 & 0.00093 & II & Kepler \\
56371.65167 & 0.00040 & I & Kepler \\
56378.25150 & 0.00085 & II & Kepler \\
56387.18835 & 0.00043 & I & Kepler \\
54983.37810 & 0.00011 & I & Kepler \\
54996.86620 & 0.01383 & II & Kepler \\
55026.64130 & 0.00008 & I & Kepler \\
55045.02337 & 0.00014 & II & Kepler \\
55069.90481 & 0.00014 & I & Kepler \\
& 5508.28656 & 0.00024 & II & Kepler \\
& & 0.00020 & I & Kepler \\
& 0.00009 & II & Kepler \\
& & & &
\end{tabular}




\begin{tabular}{|ccccc|}
\hline Times of minima: & & & \\
\hline Star name & Time of min. & Error & Type & Filter \\
& HJD 2400000+ & & & \\
\hline KIC 8378922 & 55199.69468 & 0.00013 & I & Kepler \\
& 55218.07668 & 0.00019 & II & Kepler \\
55242.95779 & 0.00007 & I & Kepler \\
& 55261.33969 & 0.00009 & II & Kepler \\
55286.22128 & 0.00006 & I & Kepler \\
55304.60317 & 0.00012 & II & Kepler \\
55329.48446 & 0.00015 & I & Kepler \\
55347.86640 & 0.00010 & II & Kepler \\
55372.74784 & 0.00017 & I & Kepler \\
55391.12985 & 0.00016 & II & Kepler \\
55416.01096 & 0.00012 & I & Kepler \\
55434.39315 & 0.00020 & II & Kepler \\
55459.27439 & 0.00007 & I & Kepler \\
55477.65611 & 0.00011 & II & Kepler \\
55502.53771 & 0.00009 & I & Kepler \\
55520.91965 & 0.00010 & II & Kepler \\
55545.80089 & 0.00012 & I & Kepler \\
55589.06433 & 0.00027 & I & Kepler \\
55607.44614 & 0.00014 & II & Kepler \\
55632.32786 & 0.00032 & I & Kepler \\
55650.70945 & 0.00012 & II & Kepler \\
55675.59088 & 0.00005 & I & Kepler \\
55693.97278 & 0.00011 & II & Kepler \\
55718.85429 & 0.00011 & I & Kepler \\
55737.23593 & 0.00013 & II & Kepler \\
55762.11735 & 0.00016 & I & Kepler \\
55780.49930 & 0.00018 & II & Kepler \\
55805.38079 & 0.00012 & I & Kepler \\
55823.76251 & 0.00015 & II & Kepler \\
55848.64406 & 0.00017 & I & Kepler \\
55867.02620 & 0.00018 & II & Kepler \\
55891.90753 & 0.00008 & I & Kepler \\
55910.28934 & 0.00011 & II & Kepler \\
55935.17062 & 0.00009 & I & Kepler \\
55953.55217 & 0.00038 & II & Kepler \\
55978.43406 & 0.00006 & I & Kepler \\
56021.69718 & 0.00011 & I & Kepler \\
56040.07918 & 0.00011 & II & Kepler \\
56064.96051 & 0.00014 & I & Kepler \\
56083.34247 & 0.00009 & II & Kepler \\
56108.22411 & 0.00021 & I & Kepler \\
& & 0.00014 & I & Kepler \\
& & & \\
& & & \\
& 56169.86905 & 0.00937 & II & Kepler \\
\hline
\end{tabular}




\begin{tabular}{|c|c|c|c|c|}
\hline \multicolumn{5}{|c|}{ Times of minima: } \\
\hline Star name & $\begin{array}{l}\text { Time of min. } \\
\text { HJD 2400000+ }\end{array}$ & Error & Туре & Filter Rem. \\
\hline \multirow[t]{10}{*}{ KIC 8378922} & 56194.75053 & 0.00011 & $\mathrm{I}$ & Kepler \\
\hline & 56213.13228 & 0.00012 & II & Kepler \\
\hline & 56256.39533 & 0.00016 & II & Kepler \\
\hline & 56281.27725 & 0.00011 & I & Kepler \\
\hline & 56299.65863 & 0.00010 & II & Kepler \\
\hline & 56324.54045 & 0.00020 & I & Kepler \\
\hline & 56342.92203 & 0.00011 & II & Kepler \\
\hline & 56367.80360 & 0.00014 & $\mathrm{I}$ & Kepler \\
\hline & 56386.18541 & 0.00013 & II & Kepler \\
\hline & 56411.06697 & 0.00029 & I & Kepler \\
\hline \multirow[t]{33}{*}{ KIC 8610483} & 54979.11435 & 0.00018 & II & Kepler \\
\hline & 54993.19590 & 0.00012 & $\mathrm{I}$ & Kepler \\
\hline & 55027.91397 & 0.00017 & II & Kepler \\
\hline & 55041.99582 & 0.00007 & I & Kepler \\
\hline & 55076.71334 & 0.00021 & II & Kepler \\
\hline & 55090.79515 & 0.00014 & $\mathrm{I}$ & Kepler \\
\hline & 55125.51268 & 0.00020 & II & Kepler \\
\hline & 55139.59424 & 0.00015 & $\mathrm{I}$ & Kepler \\
\hline & 55174.31172 & 0.00020 & II & Kepler \\
\hline & 55188.39387 & 0.00012 & I & Kepler \\
\hline & 55223.11134 & 0.00027 & II & Kepler \\
\hline & 55237.19288 & 0.00019 & $\mathrm{I}$ & Kepler \\
\hline & 55271.91040 & 0.00018 & II & Kepler \\
\hline & 55285.99261 & 0.00020 & I & Kepler \\
\hline & 55320.70922 & 0.00018 & II & Kepler \\
\hline & 55334.79185 & 0.00016 & I & Kepler \\
\hline & 55369.50901 & 0.00019 & II & Kepler \\
\hline & 55383.59097 & 0.00006 & I & Kepler \\
\hline & 55418.30833 & 0.00018 & II & Kepler \\
\hline & 55432.39064 & 0.00026 & I & Kepler \\
\hline & 55467.10674 & 0.00026 & II & Kepler \\
\hline & 55481.19033 & 0.00014 & I & Kepler \\
\hline & 55515.90680 & 0.00019 & II & Kepler \\
\hline & 55529.98911 & 0.00018 & I & Kepler \\
\hline & 55578.78863 & 0.00014 & I & Kepler \\
\hline & 55613.50558 & 0.00017 & II & Kepler \\
\hline & 55627.58824 & 0.00009 & I & Kepler \\
\hline & 55662.30479 & 0.00018 & II & Kepler \\
\hline & 55676.38754 & 0.00019 & I & Kepler \\
\hline & 55711.10417 & 0.00018 & II & Kepler \\
\hline & 55725.18684 & 0.00015 & I & Kepler \\
\hline & 55759.90356 & 0.00019 & II & Kepler \\
\hline & 55773.98581 & 0.00012 & $\mathrm{I}$ & Kepler \\
\hline
\end{tabular}




\begin{tabular}{|c|c|c|c|c|}
\hline \multicolumn{5}{|c|}{ Times of minima: } \\
\hline Star name & $\begin{array}{l}\text { Time of min. } \\
\text { HJD 2400000+ }\end{array}$ & Error & Type & Filter Rem. \\
\hline \multirow{24}{*}{ KIC 8610483} & 55808.70286 & 0.00018 & II & Kepler \\
\hline & 55822.78575 & 0.00006 & $\mathrm{I}$ & Kepler \\
\hline & 55857.50199 & 0.00021 & II & Kepler \\
\hline & 55871.58490 & 0.00012 & I & Kepler \\
\hline & 55906.30173 & 0.00026 & II & Kepler \\
\hline & 55920.38415 & 0.00020 & I & Kepler \\
\hline & 55955.10031 & 0.00731 & II & Kepler \\
\hline & 55969.18329 & 0.00011 & I & Kepler \\
\hline & 56003.90024 & 0.00023 & II & Kepler \\
\hline & 56017.98293 & 0.00016 & $\mathrm{I}$ & Kepler \\
\hline & 56052.69900 & 0.00028 & II & Kepler \\
\hline & 56066.78247 & 0.00006 & $\mathrm{I}$ & Kepler \\
\hline & 56101.49863 & 0.00216 & II & Kepler \\
\hline & 56115.58146 & 0.00007 & $\mathrm{I}$ & Kepler \\
\hline & 56150.29732 & 0.00022 & II & Kepler \\
\hline & 56164.38107 & 0.00012 & I & Kepler \\
\hline & 56199.09692 & 0.00020 & II & Kepler \\
\hline & 56213.18063 & 0.00007 & $\mathrm{I}$ & Kepler \\
\hline & 56261.97970 & 0.00008 & I & Kepler \\
\hline & 56296.69567 & 0.00016 & II & Kepler \\
\hline & 56345.49529 & 0.00019 & II & Kepler \\
\hline & 56359.57875 & 0.00015 & I & Kepler \\
\hline & 56394.29463 & 0.00028 & II & Kepler \\
\hline & 56408.37779 & 0.00024 & I & Kepler \\
\hline \multirow[t]{19}{*}{ KIC 12217907} & 54979.59790 & 0.00011 & $\mathrm{I}$ & Kepler \\
\hline & 54993.91307 & 0.00032 & II & Kepler \\
\hline & 55022.80262 & 0.00010 & I & Kepler \\
\hline & 55037.11743 & 0.00030 & II & Kepler \\
\hline & 55066.00721 & 0.00017 & I & Kepler \\
\hline & 55080.32264 & 0.00036 & II & Kepler \\
\hline & 55109.21177 & 0.00014 & I & Kepler \\
\hline & 55123.52771 & 0.00078 & II & Kepler \\
\hline & 55152.41615 & 0.00018 & $\mathrm{I}$ & Kepler \\
\hline & 55166.73097 & 0.00021 & II & Kepler \\
\hline & 55195.62084 & 0.00019 & $\mathrm{I}$ & Kepler \\
\hline & 55209.93493 & 0.00053 & II & Kepler \\
\hline & 55238.82545 & 0.00014 & I & Kepler \\
\hline & 55253.14006 & 0.00025 & II & Kepler \\
\hline & 55282.03019 & 0.00017 & I & Kepler \\
\hline & 55296.34485 & 0.00031 & II & Kepler \\
\hline & 55325.23491 & 0.00014 & I & Kepler \\
\hline & 55339.54953 & 0.00037 & II & Kepler \\
\hline & 55368.43919 & 0.00011 & I & Kepler \\
\hline
\end{tabular}




\begin{tabular}{|c|c|c|c|c|}
\hline \multicolumn{5}{|c|}{ Times of minima: } \\
\hline Star name & $\begin{array}{l}\text { Time of min. } \\
\text { HJD 2400000+ }\end{array}$ & Error & Type & Filter Rem. \\
\hline KIC 12217907 & 55382.75336 & 0.00030 & II & Kepler \\
\hline & 55411.64384 & 0.00013 & I & Kepler \\
\hline & 55425.95842 & 0.00039 & II & Kepler \\
\hline & 55454.84862 & 0.00017 & $\mathrm{I}$ & Kepler \\
\hline & 55469.16325 & 0.00037 & II & Kepler \\
\hline & 55498.05309 & 0.00009 & $\mathrm{I}$ & Kepler \\
\hline & 55512.36771 & 0.00038 & II & Kepler \\
\hline & 55541.25793 & 0.00015 & I & Kepler \\
\hline & 55584.46223 & 0.00008 & $\mathrm{I}$ & Kepler \\
\hline & 55598.77757 & 0.00059 & II & Kepler \\
\hline & 55627.66700 & 0.00013 & $\mathrm{I}$ & Kepler \\
\hline & 55641.98109 & 0.00039 & II & Kepler \\
\hline & 55670.87142 & 0.00012 & $\mathrm{I}$ & Kepler \\
\hline & 55685.18621 & 0.00026 & II & Kepler \\
\hline & 55714.07598 & 0.00015 & $\mathrm{I}$ & Kepler \\
\hline & 55728.39061 & 0.00024 & II & Kepler \\
\hline & 55757.28066 & 0.00011 & $\mathrm{I}$ & Kepler \\
\hline & 55771.59552 & 0.00023 & II & Kepler \\
\hline & 55800.48521 & 0.00014 & $\mathrm{I}$ & Kepler \\
\hline & 55814.80019 & 0.00030 & II & Kepler \\
\hline & 55843.68972 & 0.00018 & $\mathrm{I}$ & Kepler \\
\hline & 55858.00434 & 0.00039 & II & Kepler \\
\hline & 55886.89424 & 0.00016 & I & Kepler \\
\hline & 55901.20841 & 0.00039 & II & Kepler \\
\hline & 55930.09913 & 0.00013 & $\mathrm{I}$ & Kepler \\
\hline & 55944.41260 & 0.00028 & II & Kepler \\
\hline & 55973.30362 & 0.00010 & $\mathrm{I}$ & Kepler \\
\hline & 56016.50769 & 0.00021 & I & Kepler \\
\hline & 56030.82287 & 0.00024 & II & Kepler \\
\hline & 56059.71278 & 0.00016 & I & Kepler \\
\hline & 56074.02721 & 0.00038 & II & Kepler \\
\hline & 56102.91721 & 0.00011 & I & Kepler \\
\hline & 56117.23182 & 0.00018 & II & Kepler \\
\hline & 56146.12179 & 0.00010 & $\mathrm{I}$ & Kepler \\
\hline & 56160.43603 & 0.00026 & II & Kepler \\
\hline & 56189.32643 & 0.00009 & I & Kepler \\
\hline & 56203.64252 & 0.00042 & II & Kepler \\
\hline & 56232.53102 & 0.00013 & $\mathrm{I}$ & Kepler \\
\hline & 56275.73561 & 0.00016 & $\mathrm{I}$ & Kepler \\
\hline & 56290.05030 & 0.00019 & II & Kepler \\
\hline & 56333.25518 & 0.00096 & II & Kepler \\
\hline & 56362.14470 & 0.00018 & $\mathrm{I}$ & Kepler \\
\hline & 56376.45947 & 0.00021 & II & Kepler \\
\hline & 56405.34955 & 0.00008 & I & Kepler \\
\hline
\end{tabular}



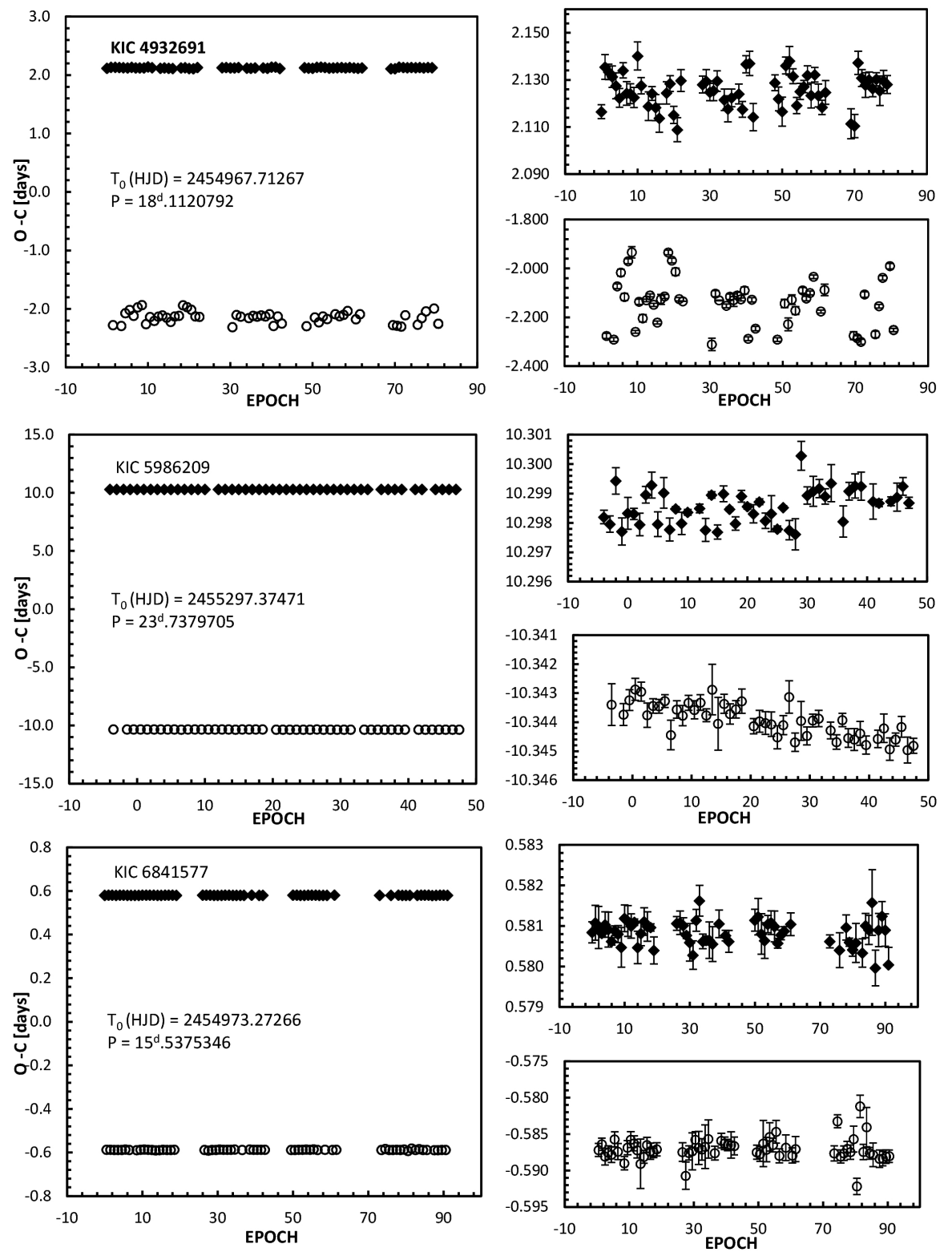

Figure 1. O-C diagrams for KIC 4932691, KIC 5986209, KIC 6841577 determined for primary and secondary minima separately (right) and together (left). The primary minima are denoted by filled symbols, the secondary minima by the symbols. 

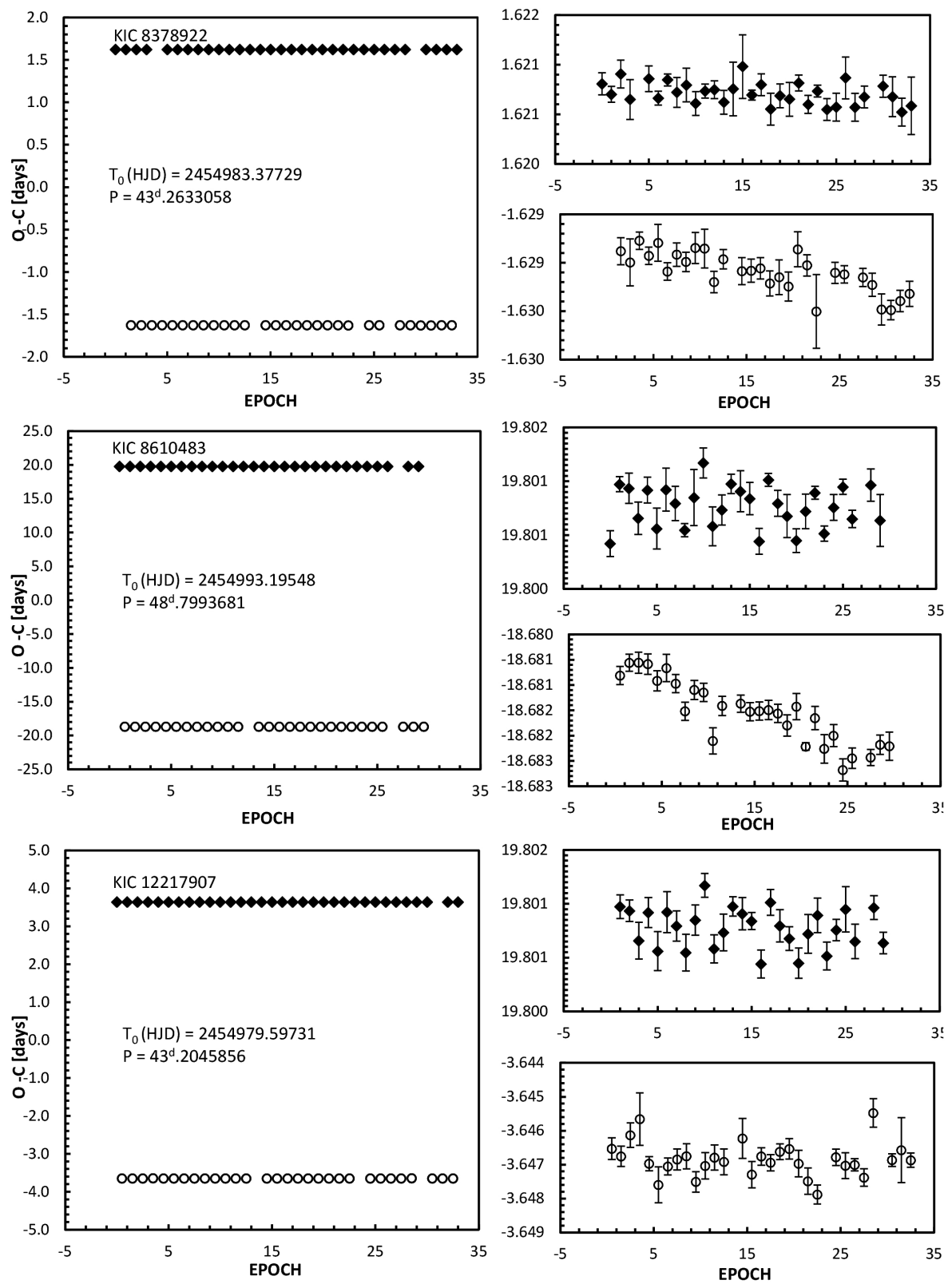

Figure 2. O-C diagrams for KIC 8378922, KIC 8610483, KIC 12217907. 